Proceedings of the

International Geometry Center

Vol. 9, no. 3-4 (2016) pp. 5-16

\title{
A new curvature-like tensor in an almost contact Riemannian manifold
}

\author{
Koji Matsumoto \\ Memories of Professor Victor Kuzakon at ONAFT
}

\begin{abstract}
In a M. Prvanović's paper [5], we can find a new curvature-like tensor in an almost Hermitian manifold.

In this paper, we define a new curvature-like tensor, named contact holomorphic Riemannian, briefly $(C H R)$, curvature tensor in an almost contact Riemannian manifold. Then, using this tensor, we mainly research $(C H R)$ curvature tensor in a Kenmotsu and a Sasakian manifold. We introduce the flatness of a $(C H R)$-curvature tensor and show that a Kenmotsu and a Sasakian manifold with a flat $(C H R)$-curvature tensor is flat, see Theorems 3.1 and 4.1. Next, we introduce the notion of an $(C H R)$ - $\eta$-Einstein in an almost contact Riemannian manifold. In particular, in a Sasakian or a Kenmotsu manifold, a $(C H R)-\eta$-Einstein manifold is $\eta$-Einstein, see Theorem 5.3. Finally, from this tensor, we introduce a notion of a $(C H R)$-space form in an almost contact Riemannian manifold. In particular, if a Kenmotsu and a Sasakian manifold are $(C H R)$-space form, then the $(C H R)$-curvature tensor satisfies a special equation, see Theorems 6.2 and 7.1.
\end{abstract}

\section{Almost contact Riemannian manifolds}

A real $(2 n+1)$-dimensional differentiable Riemannian manifold $(M, g)$ is said to be an almost contact Riemannian manifold if it has a $(1,1)$-tensor $\varphi$ and a 1 -form $\eta$ which satisfy [6]

$$
\begin{gathered}
\varphi^{2}=-I+\eta \otimes \xi, \quad \eta(\varphi X)=0, \quad \eta(\xi)=1, \\
g(\varphi X, \varphi Y)=g(X, Y)-\eta(X) \eta(Y),
\end{gathered}
$$

for any $Y, X \in T M$, where $\xi$ is defined by $g(\xi, X)=\eta(X)$, and $T M$ is the tangent bundle of $M$. From $(1.1)_{2}$ the vector field $\xi$ is a unit vector field

2010 Mathematics Subject Classification: 53C40

Keywords: curvature-like tensor, almost contact Riemannian manifold, Kenmotsu manifold, Sasakian manifold, Sasakian space form, contact holomorphic Riemannian curvature tensor 
and we call it the structure vector field of the almost contact Riemannian manifold. Next, in an almost contact Riemannian manifold $M$, we define a 2-form $F$ as $F(X, Y)=g(\varphi X, Y)$ for any $X, Y \in T M$. Then the 2-form $F$ is skew-symmetric and we call it the fundamental 2 -form of this almost contact Riemannian manifold.

In an almost contact Riemannian manifold a 2-dimensional distribution spanned by a unit vector field $X$ and $\varphi X$ is called a $\varphi$-section of $X$. The sectional curvature $R(X, \varphi X, \varphi X, X)$ is said to be the $\varphi$-holomorphic sectional curvature of $X$, where $R$ denotes the Riemannian curvature tensor with respect to $g$.

An almost contact Riemannian manifold $(M, \varphi, g, \xi)$ is called a Kenmotsu manifold, [1], [2], [3], if the structure tensors satisfy

$$
\begin{gathered}
\left(\nabla_{X} \varphi\right) Y=-\eta(Y) \varphi X-g(X, \varphi Y) \xi, \\
\nabla_{X} \xi=X-\eta(X) \xi,
\end{gathered}
$$

for any $X, Y \in T M$, where $\nabla$ means the covariant derivation with respect to $g$. In a Kenmotsu manifold $(M, \varphi, g, \xi)$ we know

$$
\begin{aligned}
\left(\nabla_{X} \eta\right) Y & =g(\varphi X, \varphi Y), \\
\eta(R(X, Y) Z) & =\eta(Y) g(X, Z)-\eta(X) g(Y, Z), \\
R(X, Y) \xi & =\eta(X) Y-\eta(Y) X, \\
R(\xi, X) Y & =\eta(Y) X-g(X, Y) \xi, \\
R_{1}(\varphi X, \varphi Y) & =R_{1}(X, Y)+2 n \eta(X) \eta(Y), \\
R_{1}(X, \xi) & =-2 n \eta(X),
\end{aligned}
$$

for any $X, Y, Z, W \in T M$, where $R_{1}$ denotes the Ricci tensor with respect to $g$.

Moreover, in a Kenmotsu manifold we get

$$
\begin{aligned}
& R(X, Y, \varphi, \varphi W)=R(X, Y, Z, W)+ \\
&+g(\varphi X, Z) g(\varphi Y, W)-g(\varphi X, W) g(\varphi Y, Z)+ \\
&+g(X, W) g(Y, Z)-g(X, Z) g(Y, W), \\
& R(X, Y, \varphi Z, W)=R(X, Y, Z, \varphi W)++g(\varphi X, W) g(Y, Z)-g(\varphi Y, W) g(X, Z)+ \\
& \quad+g(\varphi Y, Z) g(X, W)-g(\varphi X, Z) g(Y, W), \\
& R(X, Y, \varphi Z, \varphi W)=R(\varphi X, \varphi Y, Z, W), \\
& R(\varphi X, \varphi Y, \varphi Z, \varphi W)=R(X, Y, Z, W)+ \\
& \quad+\eta(X) \eta(W) g(Y, Z)-\eta(X) \eta(Z) g(Y, W)+ \\
& \quad+\eta(Y) \eta(Z) g(X, W)-\eta(Y) \eta(W) g(X, Z) .
\end{aligned}
$$


A Kenmotsu manifold with constant $\varphi$-holomorphic sectional curvature $c$ is called a Kenmotsu space form with $c$. Then, [1], its curvature tensor $R$ is expressed by

$$
\begin{aligned}
R(X, Y, Z, W) & =\frac{c-3}{4}\{g(Y, Z) g(X, W)-g(X, Z) g(Y, W)\}+ \\
+\frac{c+1}{4}[ & \{\eta(X) g(Y, W)-\eta(Y) g(X, W)\} \eta(Z)+ \\
& +\{\eta(Y) g(X, Z)-\eta(X) g(Y, Z)\} \eta(W)+ \\
& +F(Y, Z) F(X, W)-F(X, Z) F(Y, W)- \\
& -2 F(X, Y) F(Z, W)]
\end{aligned}
$$

A Kenmotsu manifold is said to be flat if its $\varphi$-sectional curvature is equal to zero on $M$.

An almost contact Riemannian manifold $(M, \varphi, g, \xi)$ is called a normal contact Riemannian or Sasakian manifold if the structure vector field $\xi$ and the fundamental 2-form $F$ satisfy

$$
\begin{gathered}
\nabla_{X} \xi=\varphi X \\
\left(\nabla_{X} F\right)(Y, Z)=\eta(Y) g(X, Z)-\eta(Z) g(X, Y)
\end{gathered}
$$

for any $X, Y, Z \in T M$.

In a Sasakian manifold the Riemannian curvature tensor $R$ with respect to $g$ satisfies

$$
\begin{aligned}
& R(X, Y, Z, \xi)=\eta(X) g(Y, Z)-\eta(Y) g(X, Z) \\
& R(X, Y, \varphi Z, W)-R(X, Y, Z, \varphi W)= \\
& =g(\varphi X, Z) g(Y, W)-g(\varphi X, W) g(Y, Z)+ \\
& \quad+g(\varphi Y, W) g(X, Z)-g(\varphi Y, Z) g(X, W), \\
& R(X, Y, \varphi Z, \varphi W)=R(\varphi X, \varphi Y, Z, W)=R(X, Y, Z, W)+ \\
& \quad+g(X, Z) g(Y, W)-g(Y, Z) g(X, W)+ \\
& \quad+F(X, W) F(Y, Z)-F(Y, W) F(X, Z), \\
& \quad R(\varphi X, \varphi Y, \varphi Z, \varphi W)=R(X, Y, Z, W)+ \\
& \quad+\eta(X) \eta(Z) g(Y, W)-\eta(X) \eta(W) g(Y, Z)+ \\
& \quad+\eta(Y) \eta(W) g(X, Z)-\eta(Y) \eta(Z) g(X, W), \\
& R_{1}(X, \xi)=2 n \eta(X)
\end{aligned}
$$

for any $X, Y, Z, W \in T M$.

A Sasakian manifold is said to be a Sasakian space form if it has constant $\varphi$-holomorpic sectional curvature. Then the curvature tensor field $R$ 
satisfies $([4])$

$$
\begin{aligned}
R(X, Y, Z, W) & =\frac{c+3}{4}\{g(X, W) g(Y, Z)-g(X, Z) g(Y, W)\}+ \\
+\frac{c-1}{4}[ & \eta(X) \eta(Z) g(Y, W)-\eta(Y) \eta(Z) g(X, W)+ \\
& +\eta(Y) \eta(W) g(X, Z)-\eta(Y) \eta(Z) g(X, W)+ \\
& +F(Y, Z) F(X, W)-F(X, Z) F(Y, W)- \\
& -2 F(X, Y) F(Z, W)]
\end{aligned}
$$

where $c$ is a constant holomorphic sectional curvature.

A Sasakian space form with zero holomorphic sectional curvature is called flat.

An almost contact Riemannian manifold $M^{2 n+1}$ is said to be $\eta$-Einstein if the Ricci tensor $R_{1}$ with respect to $g$ satisfies

$$
R_{1}(X, Y)=a g(X, Y)+b \eta(X) \eta(Y)
$$

for certain differentiable functions $a$ and $b$ on $M^{2 n+1}$ which are called the associated functions of $R_{1}$. In particular, in an $\eta$-Einstein Kenmotsu and an $\eta$-Einstein Sasakian manifold, associated functions $a$ and $b$ respectively satisfy the following relations

$$
a+b=-2 n, \quad \tau=2 n(a-1),
$$

and

$$
a+b=2 n, \quad \tau=2 n(a+1),
$$

where $\tau$ is the scalar curvature with respect to $g$.

\section{A NEW CURVATURE-LIKE TENSOR FIELD IN AN ALMOST CONTACT RIEMANNIAN MANIFOLD}

In this section, we define a new curvature-like tensor field in an almost contact Riemannian manifold.

In a differentiable manifold $M$ a $(0,4)$-type tensor field $T(X, Y, Z, W)$ is called curvature-like if it satisfies

$$
\begin{gathered}
T(X, Y, Z, W)=-T(Y, X, Z, W)=-T(X, Y, W, Z)=T(Z, W, X, Y), \\
T(X, Y, Z, W)+T(X, Z, W, Y)+T(X, W, Y, Z)=0,
\end{gathered}
$$

for any $X, Y, Z, W \in T M$. Of course, a Riemannian curvature tensor, a conformal curvature tensor in a Riemannian manifold, a Bochner curvature tensor in a Kählerian manifold and a $C$-Bochner curvature tensor in a Sasakian manifold are examples of curvature-like tensor fields. 
In an almost contact Riemannian manifold $(M, \varphi, \xi, g)$, we define a $(0,4)$ type tensor field $(C H R)(X, Y, Z, W)$ as

$$
\begin{aligned}
(C H R)(X, Y, Z, W)=\frac{1}{16}\{3[R(X, Y, Z, W)+R(\varphi X, \varphi Y, Z, W)+ \\
+ \\
+R(X, Y, \varphi Z, \varphi W)+R(\varphi X, \varphi Y, \varphi Z, \varphi W)]- \\
-R(X, Z, \varphi W, \varphi Y)-R(\varphi X, \varphi Z, W, Y)- \\
-R(X, W, \varphi Y, \varphi Z)-R(\varphi X, \varphi W, Y, Z)+ \\
+R(\varphi X, Z, \varphi W, Y)+R(X, \varphi Z, W, \varphi Y)+ \\
+R(\varphi X, W, Y, \varphi Z)+R(X, \varphi W, \varphi Y, Z)+ \\
+\eta(X)[2\{R(Z, W, Y, \xi)+R(\varphi Z, \varphi W, Y, \xi)\}+R(\varphi Z, \varphi Y, W, \xi)+ \\
\quad+R(\varphi Y, \varphi W, Z, \xi)-R(\varphi Y, W, \varphi Z, \xi)-R(Z, \varphi Y, \varphi W, \xi)]- \\
-\eta(Y)[2\{R(Z, W, X, \xi)+R(\varphi Z, \varphi W, X, \xi)\}+R(\varphi Z, \varphi X, W, \xi)+ \\
\quad+R(\varphi X, \varphi W, Z, \xi)-R(Z, \varphi X, \varphi W, \xi)-R(\varphi X, W, \varphi Z, \xi)]+ \\
+\eta(Z)[2\{R(X, Y, W, \xi)+R(\varphi X, \varphi Y, W, \xi)\}+R(\varphi X, \varphi W, Y, \xi)+ \\
\quad+R(\varphi W, \varphi Y, X, \xi)-R(\varphi W, Y, \varphi X, \xi)-R(X, \varphi W, \varphi Y, \xi)]- \\
-\eta(W)[2\{R(X, Y, Z, \xi)+R(\varphi X, \varphi Y, Z, \xi)\}+R(\varphi X, \varphi Z, Y, \xi)+ \\
\quad+R(\varphi Z, \varphi Y, X, \xi)-R(X, \varphi Z, \varphi Y, \xi)-R(\varphi Z, Y, \varphi X, \xi)]\}
\end{aligned}
$$

for any $X, Y, Z, W \in T M$. Then, by the straightforward calculation, we can check the above tensor field is curvature-like. We call this tensor field a contact holomorphic Riemannian curvature tensor in an almost contact Riemannian manifold.

A contact Riemannian manifold is said to be $(C H R)$-flat if the $(C H R)$ curvature tensor vanishes, identically.

In an almost contact Riemannian manifold the $(\mathrm{CHR})$-curvature tensor satisfies

$$
\begin{aligned}
& 16(C H R)(\varphi X, \varphi Y, Z, W)=16(C H R)(X, Y, Z, W)+ \\
&+ \eta(X)\{R(Z, W, Y, \xi)+R(\varphi Z, \varphi W, Y, \xi)\}- \\
&-\eta(Y)\{R(Z, W, X, \xi)+R(\varphi Z, \varphi W, X, \xi)\}+ \\
&+T(X, Y, Z, W), \\
& 16(C H R)(X, Y, \varphi Z, \varphi W)=16(C H R)(X, Y, Z, W)+ \\
&+\eta(Z)\{R(X, Y, W, \xi)+R(\varphi X, \varphi Y, W, \xi)\}- \\
&-\eta(W)\{R(X, Y, Z, \xi)+R(\varphi X, \varphi Y, Z, \xi)\}+ \\
&+ T(X, Y, Z, W),
\end{aligned}
$$




$$
\begin{aligned}
16(C H R)(\varphi X, & \varphi, \varphi Z, \varphi W)=16(C H R)(X, Y, Z, W)+ \\
& +\eta(X)\{R(Z, W, Y, \xi)+R(\varphi Z, \varphi W, Y, \xi)\}- \\
& -\eta(Y)\{R(Z, W, X, \xi)+R(\varphi Z, \varphi W, X, \xi)\}+ \\
& +\eta(Z)\{R(X, Y, W, \xi)+R(\varphi X, \varphi Y, W, \xi)\}- \\
& -\eta(W)\{R(X, Y, Z, \xi)+R(\varphi X, \varphi Y, Z, \xi)\}- \\
& -\eta(Z)\{\eta(X) R(\xi, Y, W, \xi)+\eta(Y) R(X, \xi, W, \xi)\}+ \\
& +\eta(W)\{\eta(X) R(\xi, Y, Z, \xi)+\eta(Y) R(X, \xi, Z, \xi)\}+ \\
& +T(X, Y, Z, W), \\
& +\eta(X H R)(X, Y, Z, \xi)=\frac{1}{16}[R(X, Y, Z, \xi)+R(\varphi X, \varphi Y, Z, \xi)+ \\
& +\eta(X)\{2 R(Y, \xi, Z, \xi)-R(\varphi Y, \xi, \varphi Z, \xi)\}- \\
& -\eta(Y)\{2 R(X, \xi, Z, \xi)-R(\varphi X, \xi, \varphi Z, \xi)\}],
\end{aligned}
$$

where we put

$$
\begin{aligned}
T(X, Y, Z, W)=\eta(Z)\{ & -2 \eta(X) R(\xi, Y, W, \xi)-2 \eta(Y) R(X, \xi, W, \xi)+ \\
& +\eta(X) R(\xi \varphi W, \varphi Y, \xi)+\eta(Y) R(\varphi W, \xi, \varphi X, \xi)\}- \\
-\eta(W)\{ & -2 \eta(X) R(\xi, Y, Z, \xi)-2 \eta(Y) R(X, \xi, Z, \xi)+ \\
& +\eta(X) R(\xi, \varphi Z, \varphi Y, \xi)+\eta(Y) R(\varphi Z, \xi, \varphi X, \xi)\} .
\end{aligned}
$$

Moreover, we have

$$
\begin{aligned}
& (C H R)(X, \varphi X, \varphi X, X)= \\
& =\frac{1}{16}[16 R(X, \varphi X, \varphi X, X)+8 \eta(X) R(X, \varphi X, \varphi X, \xi)+ \\
& \left.+\eta(X)^{2}\{11 R(\xi, \varphi X, \varphi X, \xi)+4 R(\xi, X, X, \xi)\}\right] .
\end{aligned}
$$

For a unit vector field $X$ the following tensor $(C H R)(X, \varphi X, \varphi X, X)$ is called a $\phi$-holomorphic $(C H R)$-curvature of $X$, and an almost contact Riemannian manifold is called a $(C H R)$-space form if the holomorphic $(C H R)$ curvature constant on $M$. 


\section{3. $(C H R)$-CURVAture tensor in a Kenmotsu manifold}

In this section we consider a $(C H R)$-curvature tensor in a Kenmotsu manifold. By virtue of (1.2) and (1.3) we have

$$
\begin{aligned}
& (C H R)(X, Y, Z, W)=R(X, Y, Z, W)+ \\
& +\frac{1}{4}\{g(\varphi X, Z) g(\varphi Y, W)-g(\varphi X, W) g(\varphi Y, Z)+2 g(\varphi X, Y) g(\varphi Z, W)+ \\
& \quad+\eta(X) \eta(W) g(Y, Z)-\eta(X) \eta(Z) g(Y, W)+ \\
& \quad+\eta(Y) \eta(Z) g(X, W)-\eta(Y) \eta(W) g(X, Z)\}+ \\
& +\frac{3}{4}\{g(X, W) g(Y, Z)-g(X, Z) g(Y, W)\} .
\end{aligned}
$$

Let the manifold be $(C H R)$-flat. Then we have from (3.1) that the curvature tensor $R$ is written by

$$
\begin{aligned}
& R(X, Y, Z, W)=\frac{3}{4}\{g(X, Z) g(Y, W)-g(X, W) g(Y, Z)\}+ \\
& +\frac{1}{4}\{g(\varphi X, W) g(\varphi Y, Z)-g(\varphi X, Z) g(\varphi Y, W)-2 g(\varphi X, Y) g(\varphi Z, W)+ \\
& +\eta(X) \eta(Z) g(Y, W)-\eta(X) \eta(W) g(Y, Z)+ \\
& +\eta(Y) \eta(W) g(X, Z)-\eta(Y) \eta(Z) g(X, W)\} \text {. }
\end{aligned}
$$

Comparing (3.2) and (1.4) we obtain

Theorem 3.1. A $(C H R)$-flat Kenmotum manifold is flat.

\section{4. $(C H R)$-CURVATURE TENSOR In a SASAKIAN MANifold}

In this section, we consider a $(C H R)$-flat Sasakian manifold and prove that this manifold is a Sasakian space form with zero holomorphic sectional curvature.

By virtue of (1.5), (1.6), (1.7) and Bianchi identity, [6], the $(C H R)$ curvature tensor $(\mathrm{CHR})$ in a Sasakian manifold satisfies

$$
\begin{aligned}
(C H R)(X, Y, Z, W)=R(X, Y, Z, W)+ \\
+\frac{3}{4}\{g(X, Z) g(Y, W)-g(X, W) g(Y, Z)\}+ \\
+\frac{1}{4}\{g(\varphi X, W) g(\varphi Y, Z)-g(\varphi X, Z) g(\varphi Y, W)-2 g(\varphi X, Y) g(\varphi Z, W)+ \\
\quad+\eta(X) \eta(Z) g(Y, W)-\eta(X) \eta(W) g(Y, Z)+ \\
\quad+\eta(Y) \eta(W) g(X, Z)-\eta(Y) \eta(Z) g(X, W)\}
\end{aligned}
$$


Consider the $(C H R)$-flat case in a Sasakian manifold. Then we have from (3.1) that the curvature tensor $R$ is written as

$$
\begin{aligned}
R(X, Y, Z, W) & =\frac{3}{4}\{g(X, W) g(Y, Z)-g(X, Z) g(Y, W)\}- \\
-\frac{1}{4}\{ & g(\varphi X, W) g(\varphi Y, Z)-g(\varphi X, Z) g(\varphi Y, W)-2 g(\varphi X, Y) g(\varphi Z, W)+ \\
& +\eta(X) \eta(Z) g(Y, W)-\eta(X) \eta(W) g(Y, Z)+ \\
& +\eta(Y) \eta(W) g(X, Z)-\eta(Y) \eta(Z) g(X, W)\} .
\end{aligned}
$$

Comparing the above equation and (1.3) we get

Theorem 4.1. A (CHR)-flat Sasakian manifold is flat.

5. The properties of the $(C H R)$-Curvature tensor

In this section we consider other properties of the $(C H R)$-curvature tensor in Kenmotsu and Sasakian manifolds.

Since the $(C H R)$-curvature tensor in a Kenmotsu manifold satisfies (3.1), using (1.3) and (2.1), we obtain

Proposition 5.1. In a Kenmotsu manifold we have

$$
\begin{aligned}
& (C H R)(X, Y, Z, \xi)=0, \\
& (C H R)(X, Y, \varphi Z, \varphi W)=(C H R)(\varphi X, \varphi Y, Z, W)= \\
& \quad=(C H R)(\varphi X, \varphi Y, \varphi Z, \varphi W)=(C H R)(X, Y, Z, W), \\
& (C H R)(X, \varphi X, \varphi X, X)=R(X, \varphi X, \varphi X, X)+ \\
& \quad+\eta(X)^{2}\left\{g(X, X)-\eta(X)^{2}\right\}
\end{aligned}
$$

Similarly, we have from (1.7) and (4.1)

Proposition 5.2. In a Sasakian manifold, we have

$$
\begin{aligned}
& (C H R)(X, Y, Z, \xi)=0, \\
& (C H R)(X, Y, \varphi Z, \varphi W)=(C H R)(\varphi X, \varphi Y, Z, W)= \\
& \quad=(C H R)(\varphi X, \varphi Y, \varphi Z, \varphi W)=(C H R)(X, Y, Z, W) \\
& (C H R)(X, \varphi X, \varphi X, X)=R(X, \varphi X, \varphi X, X)- \\
& \quad-\eta(X)^{2}\left\{g(X, X)-\eta(X)^{2}\right\} .
\end{aligned}
$$

Next, we put

$$
\rho(C H R)(X, Y)=\sum_{i=1}^{2 n+1}(C H R)\left(e_{i}, X, Y, e_{i}\right)
$$


for a local orthonormal frame $\left(e_{1}, e_{2}, \ldots, e_{2 n+1}\right)$ of an almost contact Riemannian manifold $M^{2 n+1}$. We say this $\rho(C H R)(X, Y)$ a $(C H R)$-Ricci tensor. By virtue of (3.1) and (4.1), the $(C H R)$-Ricci tensor in a Kenmotsu and a Sasakian manifold are respectively written by

$$
\begin{aligned}
& \rho(C H R)(X, Y)=R_{1}(X, Y)+ \\
& +\frac{1}{2}\{(3 n-1) g(X, Y)+(n+1) \eta(X) \eta(Y)\},
\end{aligned}
$$

and

$$
\begin{aligned}
\rho(C H R)(X, Y)=R_{1}(X, Y)- \\
-\frac{1}{2}\{(3 n-1) g(X, Y)+(n+1) \eta(X) \eta(Y)\} .
\end{aligned}
$$

We also put

$$
\tau(C H R)=\sum_{i=1}^{2 n+1}(C H R)\left(e_{i}, e_{i}\right)
$$

which is called the $(C H R)$-scalar curvature of $(C H R)$-curvature tensor. Then, in a Kenmotsu and a Sasakian manifold, we respectively have from (5.3) and (5.4)

$$
\begin{aligned}
& \tau(C H R)=\tau+n(3 n+1), \\
& \tau(C H R)=\tau-n(3 n+1) .
\end{aligned}
$$

Now, a contact Riemannian manifold $M^{2 n+1}$ is called $(C H R)-\eta$-Einstein if its (CHR)-Ricci tensor $\rho(C H R)(X, Y)$ has the form

$$
\rho(C H R)(X, Y)=\alpha g(X, Y)+\beta \eta(X) \eta(Y)
$$

for certain functions $\alpha$ and $\beta$ which are called associated functions of $\rho(C H R)$. In particular, if our manifold is Kenmotsu or Sasakian, then we have from (5.3) and (5.4) the Ricci tensors are respectively written as

$$
\begin{aligned}
& R_{1}(X, Y)=\left(\alpha-\frac{3 n-1}{2}\right) g(X, Y)+\left(\beta-\frac{n+1}{2}\right) \eta(X) \eta(Y), \\
& R_{1}(X, Y)=\left(\alpha+\frac{3 n-1}{2}\right) g(X, Y)+\left(\beta+\frac{n+1}{2}\right) \eta(X) \eta(Y) .
\end{aligned}
$$

Conversely, if our manifold $M^{2 n+1}$ is $\eta$-Einstein, then we can easily that a Kenmotsu and a Sasakian manifolds are $(C H R)$ - $\eta$-Einstein. Moreover, we have from (1.9), (5.5) and (5.6)

$$
\begin{array}{ll}
\alpha+\beta=4 n & : \text { Kenmotsu, } \\
\alpha+\beta=0 & : \text { Sasakian. }
\end{array}
$$

By virtue of (5.3), (5.4), (5.8), and (5.9) the scalar curvature $\tau$ and the $(C H R)$-scalar curvature $\tau(C H R)$ are respectively written as

$$
\tau=2 n \alpha-3 n(n-1), \quad \tau(C H R)=2 n(\alpha+2) \quad: \text { Kenmotsu, }
$$




$$
\tau=2 n(\alpha+3 n+1), \quad \tau(C H R)=n(2 \alpha+3 n+1) \quad: \text { Sasakian } .
$$

Thus we have

Theorem 5.3. A Kenmotsu or a Sasakian manifold $M^{2 n+1}$ is $(C H R)-\eta$ Einstein if and only if $M^{2 n+1}$ is $\eta$-Einstein and the scalar curvatures $\tau$ and $\tau(C H R)$ are respectively written by (5.10) and (5.11) which includes only the associated function $\alpha$.

Corollary 5.4. In an (CHR)- $\eta$-Einstein Kenmotsu or Sasakian manifold $M^{2 n+1}$, the scalar curvatures $\tau$ and $\tau(C H R)$ are constant if and only if one of the associated functions is constant.

\section{6. $(C H R)$-SPACE FORM}

In this section we define a $(C H R)$-space form in an almost contact Riemannian manifold.

Let $M^{2 n+1}$ be an $(2 n+1)$-dimensional almost contact Riemannian manifold.

Definition 6.1. An almost contact Riemannian manifold $M^{2 n+1}$ is said to be a $(C H R)$-space form if its contact holomorphic $(C H R)$-sectional curvature is constant for any vector fields and any point on $M^{2 n+1}$

$$
(C H R)(X, \varphi X, \varphi X, X)=c\|X\|^{2}\left\{\|X\|^{2}-\eta(X)^{2}\right\}
$$

for a certain constant $c$ and any $X \in T M$, where $\|X\|$ is the length of $X$ with respect to $g$.

$$
(C H R)(X, \varphi X, \varphi X, X)=c\|X\|^{4}
$$

for any $X \in T M-\{\xi\}$.

First, we consider a Kenmotsu $(C H R)$-space form, that is a manifold $M$ whose $(C H R)$-curvature tensor satisfies (6.1).

By virtue of (5.1)

$$
c\|X\|^{4}=R(X, \varphi X, \varphi X, X)+\eta(X)^{2}\left\{g(X, X)-\eta(X)^{2}\right\}
$$

for any $X \in T M$. Moreover, from the above equation we know

$$
c\|X\|^{4}=R(X, \varphi X, \varphi X, X)
$$

for any $X \in T M-\{\xi\}$. This means that the manifold is a Kenmotsu space form. So, using (3.1) and Proposition 5.1, the curvature tensor $R$ is written 
as

$$
\begin{gathered}
(C H R)(X, Y, Z, W)=\frac{1}{4}\{g(X, Z) g(Y, W)-g(X, W) g(Y, Z)+ \\
+g(\varphi X, Z) g(\varphi Y, W)-g(\varphi X, W) g(\varphi Y, Z)+2 g(\varphi X, Y) g(\varphi Z, \varphi W)+ \\
+\eta(X) \eta(W) g(Y, Z)-\eta(X) \eta(Z) g(Y, W)+\eta(Y) \eta(Z) g(X, W)- \\
-\eta(Y) \eta(W) g(X, Z)\} .
\end{gathered}
$$

From the above equation, we obtain

$$
\rho(C H R)(X, Y)=-\frac{n+1}{2}\{g(X, Y)-\eta(X) \eta(Y)\}
$$

and

$$
\tau(C H R)=-n(n+1)
$$

Summing up, we have

Theorem 6.2. If an $(2 n+1)$-dimensional $(C H R)$-Kenmotsu space form, then the $(C H R)$-curvature tensor, the $(C H R)$-Ricci tensor and the $(C H R)$ scalar curvature are respectively given by (6.2), (6.3), and (6.4).

\section{SaSAKIAN $(C H R)$-SPACE FORMS}

In this section, we consider a Sasakian $(C H R)$-space form and we determine the Riemannian curvature tensor of this manifold.

Let $M$ be a Sasakian manifold. Then the $(C H R)$-curvature tensor satisfies (4.1). From this we have

$$
\begin{aligned}
(C H R)(X, \varphi X, \varphi X, X)=R(X, \varphi X, \varphi X, X)- & \\
& -\eta(X)^{2} g(X, X)+\eta(X)^{4} .
\end{aligned}
$$

Now suppose that $M$ is a Sasakian $(C H R)$-space form. Then, we have from (6.1) and (7.1) that

$$
R(X, \varphi X, \varphi X, X)=c\|X\|^{4}+\eta(X)^{2} g(X, X)-\eta(X)^{4}
$$

for any $X \in T M$. In particular, for $X \in T M-\{\xi\}$ the above equation means

$$
R(X, \varphi X, \varphi X, X)=c\|X\|^{4}
$$


that is, the manifold is a Sasakian space form with the constant $\varphi$-holomorphic sectional curvature $c$. So, its curvature tensor $R(X, Y, Z, W)$ satisfies (1.8). Substituting (1.8) into (4.1), we get

$$
\begin{aligned}
(C H R) & (X, Y, Z, W)=\frac{c}{4}\{g(X, W) g(Y, Z)-g(X, Z) g(Y, W)+ \\
& +\eta(X) \eta(Z) g(Y, W)-\eta(Y) \eta(Z) g(X, W)+\eta(Y) \eta(W) g(X, Z)- \\
& -\eta(X) \eta(W) g(Y, Z)-g(\varphi X, W) g(\varphi Y, Z)+ \\
& +g(\varphi X, Z) g(\varphi Y, W)-2 g(\varphi X, Y) g(\varphi Z, W)\} .
\end{aligned}
$$

From (7.2), we obtain

$$
\rho(C H R)(X, Y)=\frac{(n+1) c}{2}\{g(X, Y)-\eta(X) \eta(Y)\}
$$

and

$$
\tau(C H R)=n(n+1) c .
$$

As a result, we have

Theorem 7.1. If $M$ is an $(2 n+1)$-dimensional Sasakian $(C H R)$-space form, then its $(C H R)$-curvature tensor, the Ricci $(C H R)$-tensor and the scalar $(C H R)$-curvature are respectively given by (7.2), (7.3), and (7.4).

\section{REREFENCES}

[1] K. Arslan, R. Ezentas, I. Mihai, C. Murathan, C. Ozgur. Ricci curvature of submanifolds in Kenmotsu space forms. International Journal of Mathematics and Mathematical Sciences, 29(12):719-726, 2002.

[2] Avik De. On Kenmotsu manifold. Bulletin of Mathematical Analysis and Applications, $2(3): 1-6,2010$.

[3] Katsuei Kenmotsu. A class of almost contact Riemannian manifolds. Tohoku Mathematical Journal, 24(1):93-103, 1972.

[4] Koji Matsumoto, Ion Mihai. Ricci tensor of $c$-totally real submanifolds in Sasakian space forms. Nihonkai Mathematical Journal, 13(2):191-198, 2002.

[5] Mileva Prvanovic. Conformally invariant tensors of an almost Hermitian manifold associated with the holomorphic curvature tensor. Journal of Geometry, 103(89):89-101, 2012.

[6] Kentaro Yano, Masahiro Kon. Structures on Manifolds, volume 3 of Series in pure mathematics. World Scientific, 1984.

Received: November 30, 2016, accepted: January, 9, $201 \%$.

Koji Matsumoto

2-3-65 Nishi-Odori, Yonezawa, Yamagata, 992-0059, Japan

Email: tokiko_matsumoto@yahoo.com 